\title{
Universiteit
}

Leiden

The Netherlands

\section{Development of the CT Syndesmophyte Score (CTSS) in patients with ankylosing spondylitis: data from the SIAS cohort}

Bruin, F. de; Koning, A. de; Berg, R. van den; Baraliakos, X.; Braun, J.; Ramiro, S.; ... ; Heijde, D. van der

\section{Citation}

Bruin, F. de, Koning, A. de, Berg, R. van den, Baraliakos, X., Braun, J., Ramiro, S., ... Heijde, D. van der. (2018). Development of the CT Syndesmophyte Score (CTSS) in patients with ankylosing spondylitis: data from the SIAS cohort. Annals Of The Rheumatic Diseases, 77(3). doi:10.1136/annrheumdis-2017-212553

Version: $\quad$ Not Applicable (or Unknown)

License: $\quad$ Leiden University Non-exclusive license

Downloaded from: https://hdl.handle.net/1887/87138

Note: To cite this publication please use the final published version (if applicable). 
Development of the Computed Tomography Syndesmophyte Score (CTSS) in patients with Ankylosing Spondylitis: data from the SIAS cohort

F. de Bruin ${ }^{1 *}$, A. de Koning ${ }^{2 *}$, R. van den Berg², X. Baraliakos ${ }^{3}$, J. Braun ${ }^{3}$, F.A. van Gaalen², M.

Reijnierse ${ }^{1}$, D. van der Heijde ${ }^{2}$

*Authors contributed equally

${ }^{1}$ Leiden University Medical Center, department of Radiology, Leiden, the Netherlands

2Leiden University Medical Center, department of Rheumatology, Leiden, the Netherlands

${ }^{3}$ Rheumazentrum Ruhrgebiet Herne, department of Rheumatology, Herne, Germany

Corresponding author: D. van der Heijde, Leiden University Medical Center, department of Rheumatology, PO box 9600, 2300 RC Leiden, the Netherlands; mail@dvanderheijde.nl

Word count abstract (max 250): 250

Word count article ( $\max 3000): 3000$

Number of tables and figures ( $\max 6)$ ): 4 figures, 2 tables

Number of supplemental tables and figures: 0 
Abstract

Objectives: To develop the Computed Tomography Syndesmophyte Score (CTSS) for low-dose CT (IdCT) to assess structural damage in the spine of Ankylosing Spondylitis (AS) patients and test its reliability.

Methods: AS patients in the SIAS-cohort had whole spine IdCT at baseline and two years.

Syndesmophytes were scored in coronal and sagittal planes in eight quadrants per vertebral unit (VU) as absent $=0,<50 \%$ of the intervertebral disc space (IDS) $=1, \geq 50 \%=2$ or bridging the IDS $=3$ (range 0 552). Images were scored by two readers, paired by patient, blinded to time order. Whole spine and spinal segment status and change scores were calculated. Interreader reliability was assessed by ICC, smallest detectable change (SDC) and frequency of scores per VU.

Results: 49 patients (mean age 50 years (SD 9.8), 84\% male, 88\% HLA-B27 positive) were included. Mean (SD) scores of reader 1 were: whole spine baseline status score 163 (126) and change score 16 (21), spinal segment baseline status scores 30 (41), 97 (77) and 36 (36) and change scores 2 (7), 12 (18) and 3 (4) for the cervical, thoracic and lumbar spine, respectively. Scores of reader 2 were similar. Whole spine status score ICC was 0.99 and $0.97-0.98$ for spinal segments. Whole spine change score ICC was 0.77 and $0.32-0.75$ for spinal segments. Whole spine SDC was 14.4 . Score distribution pattern per VU was similar between readers.

Conclusions: Using the CTSS, syndesmophytes in the spine of AS patients are assessed with excellent interreader reliability. Most structural damage was seen in the thoracic spine.

Keywords: Ankylosing Spondylitis, spine, computed tomography, syndesmophytes, bone proliferation, structural damage, scoring method, reliability 


\section{Introduction}

Ankylosing spondylitis (AS) is a disease characterized by the formation of syndesmophytes. Currently, bone formation is assessed on lateral conventional radiographs (CRs) of the cervical and lumbar spine using the modified Stoke Ankylosing Spondylitis Spine Score (mSASSS).[1] With CR, only the anterior vertebral corners are assessed and it is not possible to reliably assess all thoracic vertebrae due to overprojection of soft tissues and bones. Furthermore, level C7 is often poorly visible due to overprojection of the shoulders. Moreover, sensitivity to change on radiographs is limited. Consequently, the minimum duration of follow up is two years to reliably assess progression in a substantial proportion of patients.[2]

Using Magnetic Resonance Imaging (MRI), multiplanar scans of the whole spine can be made. However, due to the lack of signal associated with calcification, and the similar low signal of the annulus fibrosis and ligaments, this method is less useful for the evaluation of bone formation.

Computed Tomography (CT) is the most appropriate method for assessing new bone formation, however its high radiation dose limits its use in follow up studies. But, with the development of new hardware and software, most notably the multislice scanners, iterative reconstructions and progress in imaging protocols, low dose CT (IdCT) might be a viable alternative for spinal radiographs. Major advantages of CT are the lack of overprojection and the possibility to assess the whole spine. CT has previously been used for the assessment of syndesmophytes in AS using a computer-based algorithm.[3,4] This algorithm has however only been developed for assessment of lumbar vertebrae and has only been tested on high-resolution CT-scans, which have a radiation dose of approximately $8 \mathrm{mSv}$ per lumbar-scan. It therefore seems unlikely that this method will be feasible for the assessment of the whole spine. We therefore developed a scoring method for the assessment of

syndesmophytes in the spine of AS patients on IdCT, the CT Syndesmophyte Score (CTSS). Here we describe the development and initial results.

\section{Methods}

\section{Study population and protocol}

Data from the Sensitive Imaging in Ankylosing Spondylitis (SIAS) study, an observational two year follow up study of AS patients, was used. 60 patients were included, 30 patients in the Netherlands and 30 in Germany. Inclusion criteria were a clinical diagnosis of AS, fulfillment of the modified New York criteria, 1-18 syndesmophytes (maximum of $75 \%$ of the possible sites) visible on lateral cervical and lumbar spine CR and $\geq 1$ inflammatory lesion on STIR MRI of the whole spine.[5] All treatment was allowed according to the treating rheumatologist and no specific level of disease activity was required. Exclusion criteria were pregnancy and circumstances which would invalidate informed consent or limit the ability of the patient to comply with protocol requirements. Clinical data and MRI of the whole spine were collected at baseline, one and two years. Additionally, CR of the lateral cervical and lumbar spine and whole spine IdCT were performed at baseline and two years. Patients underwent spinal imaging using a 64-section CT scanner (Aquilion 64; Toshiba Medical Systems, 
Otawara, Japan in Leiden and Somatom Emotion 16; Siemens, Erlangen, Germany in Herne).

Patients were placed in supine position, feet first. For the spine, 1-mm and 3-mm slices were acquired from the superior endplate of $\mathrm{C} 2$ down to the inferior endplate of $\mathrm{S} 1(120 \mathrm{kVp}, 60 \mathrm{~mA}, 0.5 \mathrm{~s}, 53 / 64$ pitch). The effective radiation dose per IdCT was approximately $4 \mathrm{mSv}$. The assessment of effective radiation dose per IdCT was achieved according to the methodology described in Teeuwisse et al. [6] Sagittal and coronal reconstructions were made. For the present study patients were included if IdCT was present at baseline and 2 years and if $\geq 75 \%$ of the vertebral sites that can be assessed on a IdCT were present. The medical ethical committee of both participating centers granted approval and written informed consent was obtained from all patients before inclusion.

\section{Development of CTSS}

An IdCT consists of a series of cross-sectional images (slices), allowing the viewer to "scroll through the spine" as if it were a 3D object (fig 1A). By scrolling through sagittal images, the reader is able to assess the anterior and posterior rim of the vertebral bodies. In the coronal plane, the reader can assess the left and right rim.

The spine was divided into VUs. A VU consists of the bottom half of a vertebra, the intervertebral disc space (IDS) and the top half of the next vertebra (fig 1B). From the bottom half of $\mathrm{C} 2$ to the top half of $\mathrm{S} 1$, there are $23 \mathrm{VUs}$. By scoring images in the sagittal and coronal planes, 8 quadrants are assessed per VU. In each quadrant, the reader assesses the presence of a syndesmophyte. A syndesmophyte is defined as a bony spur arising from the vertebral body close to the vertebral endplate in a vertical configuration, with a minimum angle of 45-degrees with the endplate. Syndesmophytes are scored using a method based on the mSASSS (fig 1C-E). The score reflects the height of a syndesmophyte relative to the intervertebral disc space (IDS). For every quadrant, the score is 0 if no syndesmophyte is present, 1 if a syndesmophyte is present that does not reach $50 \%$ of the IDS, 2 if the syndesmophytes reaches or crosses $50 \%$ of the IDS and 3 if the syndesmophyte bridges the IDS. A value of 3 is per definition scored in both quadrants on opposite sides of the IDS. With 8 quadrants per VU, $23 \mathrm{VUs}$ per spine and a maximum score of 3 per quadrant, the maximum possible score for a patient is 552 .

IdCT images were assessed using the CTSS by two trained readers, paired by patient, blinded to time order and patient information.

\section{$\underline{\text { Score analysis }}$}

For patients that were included in the analysis, missing scores were imputed using a previously described adaptation of the last-observation-carried-forward method.[7] Briefly, for two-year missing quadrant-scores, the mean spinal segment progression score (i.e. based on the present quadrants in the same segment) was added to the baseline quadrant-score, with a maximum of 3 . Similarly, for baseline missing quadrant-scores, the mean spinal segment progression score was subtracted from the two-year quadrant-score, with a minimum of 0 . If a quadrant-score was missing at both timepoints, the average spinal score per timepoint was used for that quadrant. Status scores were calculated by 
adding the scores of all quadrants per patient, per timepoint. Progression scores were calculated by subtracting the baseline status score from the two-year status score. This was done for the whole spine and per segment (cervical, thoracic and lumbar). The number of patients with a change score below the negative value of $0,0.5$ and the smallest detectable change (SDC) were subtracted from the number with a change score above these values to calculate the net number of patients with progression.

Analysis of the frequency of the scores 1,2 and 3 was performed by counting the number of quadrants with a certain score per $\mathrm{VU}$, for all patients combined, and dividing this by the total number of quadrants in that VU. The number of quadrants with an increase in score of $\geq 1$ between baseline and two years were counted for the whole spine. Subsequently, the number of new syndesmophytes that developed were counted for the whole spine and per segment and were related to the number of quadrants with an increase in score. The percentage of VUs with progression was calculated by counting the number VUs with a higher two-year score than the baseline score and dividing this by total number of VUs for all patients combined.

In order to assess on which side and in which VU most syndesmophytes were present at baseline, the number of syndesmophytes per side of a VU (anterior, posterior, left and right) were counted for all patients. Every side of a VU incorporates two quadrants per patient. Subsequently, the total number of available quadrants per side was calculated by multiplying the number of quadrants per side of a VU (2) with the total number of VUs per patient (23) and the number of patients (=46 available quadrants per side for all VUs and patients). Next, the total number of syndesmophytes per side of a VU was expressed as the percentage of quadrants affected by a syndesmophyte relative to the total number of quadrants that could be affected by syndesmophytes.

\section{Statistical analysis}

Patient characteristics and CTSS results were analyzed using descriptive statistics. Interobserver reliability was assessed using the SDC and the intraclass correlation coefficient (ICC).[8] The SDC is the smallest change that can be detected in an individual patient beyond measurement error and was calculated by $S D C=1.96^{*} S D_{\text {diff }} /\left(\sqrt{ } k^{*} \sqrt{2}\right)$. SD is the standard deviation of the difference in raw progression scores between two readers; $k$ is the number of readers. ICC was calculated for the whole spine and for spinal segments. Furthermore, using a heatmap, the frequency of the scores 1,2 and 3 were compared per VU between readers. Analyses were performed using STATA SE version 14 (StataCorp LP) and R version 3.4 (R Foundation). 


\section{Results}

From the 60 patients included in the SIAS cohort, 11 were excluded due to IdCT missing at two years $(n=9)$, one timepoint not scored by one reader $(n=1)$ or $>25 \%$ missing quadrants due to blurred low quality images $(n=1)$. Therefore, 49 patients were included in the analyses.

Baseline demographics and clinical characteristics are summarized in table 1. 84\% was male, mean age at inclusion was 50 years (SD 9.8), 88\% was HLA-B27 positive, mean ASDAS was 2.5 (SD 1.2), mean number of syndesmophytes at baseline on conventional radiography was 5.7 (SD 4.4, range 014) and 6.3 (SD 5.1, range 0-18) according to reader 1 and 2, respectively.

Table 1. Baseline characteristics of patients included in the analyses $(n=49)$

\begin{tabular}{ll}
\hline & Patients \\
$\mathbf{n = 4 9}(\%)$
\end{tabular}

Results are presented in number (\%) unless otherwise specified.

HLA-B27: Human Leukocyte Antigen B27, IBD: inflammatory bowel disease, CRP: C reactive protein, BASDAl: Bath Ankylosing Spondylitis Disease Activity Index, ASDAS: Ankylosing Spondylitis Disease Activity Score, CR conventional radiography of lateral cervical and lumbar spine; NSAID: NonSteroidal Anti-Inflammatory Drugs, csDMARD: conventional synthetic Disease Modifying Antirheumatic drugs. At two years 26 patients used NSAIDs, 9 csDMARDS and 10 TNF-alpha blockers

Table 2 shows the mean and median status scores per timepoint and the change scores for the whole spine and segments of the spine. Mean CTSS baseline status score for the whole spine for reader 1 was 163 (SD 126), median 127 (range 11-428). Mean change score was 16 (SD 21), median 10 (range -11-96). There was a large spread in scores with almost the entire scoring range used. Most structural damage and bone proliferation were seen in the thoracic spine with mean baseline status scores of 30 (SD 41), 97 (SD 77) and 36 (SD 36) and mean change scores of 2 (SD 7), 12 (SD 18) and 3 (SD 4) for the cervical, thoracic and lumbar spine respectively. Results were similar for reader 2. The ICC for the baseline status score was 0.99 for the whole spine and $0.97-0.98$ for spinal segments. The ICC for the change scores was somewhat lower with 0.77 for the whole spine and $0.57,0.75$ and 0.32 for the cervical, thoracic and lumbar spine respectively. The SDC was 14.4 for the whole spine, 9.3 for the cervical spine, 12.5 for the thoracic spine and 4.7 for the lumbar spine. 
Table 2. Status scores, change scores and ICC of baseline and two years for the whole spine and segments of the spine for the $\mathbf{4 9}$ patients included in the analysis.

\begin{tabular}{|c|c|c|c|c|}
\hline Timepoint & & Reader 1 & Reader 2 & ICC (95\% Cl) \\
\hline Whole spine (m & $x$ score 552 ) & & & \\
\hline Baseline & Mean (SD) & $163(126)$ & $158(124)$ & $0.99(0.98-0.99)$ \\
\hline & Median (range) & $127(11-428)$ & $115(5-445)$ & \\
\hline 2 years & Mean (SD) & $180(130)$ & $175(130)$ & $0.99(0.98-0.99)$ \\
\hline & Median (range) & $157(14-455)$ & $125(7-459)$ & \\
\hline Change score & Mean (SD) & $16(21)$ & $17(21)$ & $0.77(0.63-0.86)$ \\
\hline & Median (range) & $10(-11-96)$ & $10(-6-106)$ & \\
\hline Cervical spine & ax score 144) & & & \\
\hline Baseline & Mean (SD) & $30(41)$ & $28(37)$ & $0.98(0.96-0.99)$ \\
\hline & Median (range) & $8(0-140)$ & $8(0-134)$ & \\
\hline 2 years & Mean (SD) & $32(42)$ & $32(40)$ & $0.95(0.92-0.97)$ \\
\hline & Median (range) & $12(0-140)$ & $10(0-135)$ & \\
\hline Change score & Mean (SD) & $2(7)$ & $4(8)$ & $0.57(0.35-0.73)$ \\
\hline & Median (range) & $0(-12-39)$ & $0(-9-39)$ & \\
\hline Thoracic spine & ax score 264) & & & \\
\hline Baseline & Mean (SD) & $97(77)$ & $95(77)$ & $0.98(0.96-0.99)$ \\
\hline & Median (range) & $77(1-253)$ & $72(0-264)$ & \\
\hline 2 years & Mean (SD) & $109(80)$ & $106(81)$ & $0.99(0.98-0.99)$ \\
\hline & Median (range) & $85(1-256)$ & $78(0-264)$ & \\
\hline & Mean (SD) & $12(18)$ & $10(18)$ & $0.75(0.60-0.85)$ \\
\hline Change score & Median (range) & $6(-6-97)$ & $6(-12-93)$ & \\
\hline Lumbar spine ( & ax score 144) & & & \\
\hline Baseline & Mean (SD) & $36(36)$ & $35(35)$ & $0.97(0.95-0.98)$ \\
\hline & Median (range) & $24(0-140)$ & $22(0-140)$ & \\
\hline 2 years & Mean (SD) & $39(38)$ & $38(36)$ & $0.96(0.93-0.98)$ \\
\hline & Median (range) & $26(0-144)$ & $29(0-144)$ & \\
\hline Change score & Mean (SD) & $3(4)$ & $3(4)$ & $0.32(0.04-0.55)$ \\
\hline & Median (range) & $2(-7-13)$ & $1(-5-15)$ & \\
\hline
\end{tabular}

Table 3 presents the number of patients showing a change (positive, negative or net) according the cut-off levels 0, 0.5 and SDC. When looking at any net change for the whole spine, more than half of the patients ( $61 \%$ according to reader 1 and $76 \%$ according to reader 2 ) show a positive change. When analyzing separate spinal segments, net more patients showed a positive change in the thoracic spine compared with the lumbar and cervical spine (63\% vs $49 \%$ and $55 \%$ according to reader 1). These numbers were similar for the cut-off of 0.5 . Using the SDC as the cut-off, net $43 \%$ and $37 \%$ showed a positive change according to reader 1 and 2 respectively, with most patients showing progression in the thoracic spine. For the cervical spine, reader 1 scored 2 patients as having a change larger than the negative value of the SDC. In the lumbar spine, this occurred for 1 patient according to both readers. This did not occur for the whole or thoracic spine. 
Table 3. Number of patients with progression between baseline and two years for the whole spine and segments of the spine for the 49 patients included in the analysis.

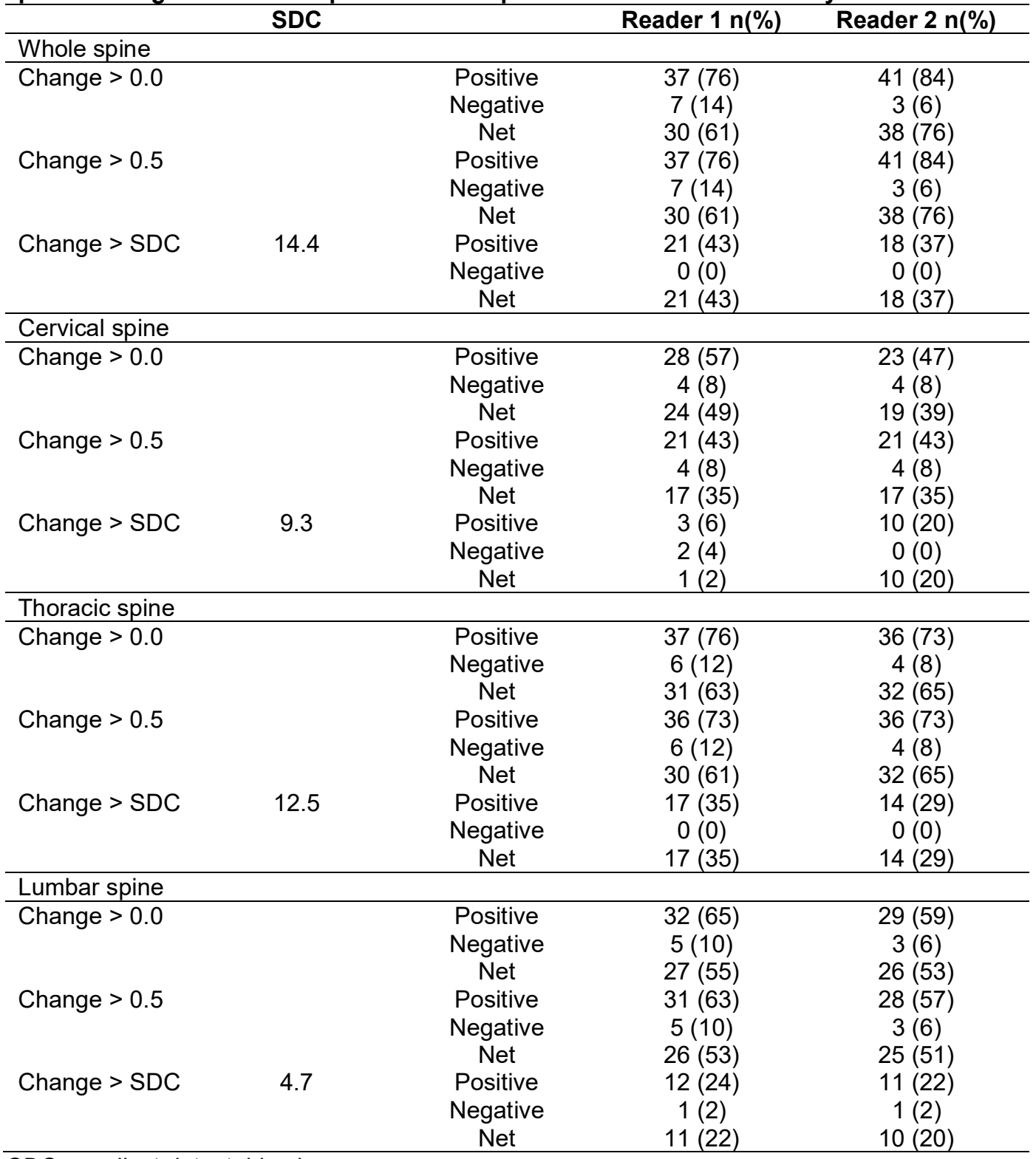

SDC: smallest detectable change

The distribution pattern of the scores 1,2 and 3 at baseline and the percentage of VUs with progression for both readers are presented in figure 2. The distribution patterns of scores and percentage of VUs with progression are very similar between readers. A score of 3 is most prevalent in the majority of the VUs. This is most obvious in the thoracic spine, where up to $45 \%$ of VUs have a score of 3 . A score of 2 is most prevalent in the lumbar spine. The highest percentage of VUs with progression is found in the thoracic spine. 
Figure 3 presents the percentage of quadrants per side of a $\mathrm{VU}$ affected by syndesmophytes relative to the total number of quadrants per side for all VUs and patients. For all sides, more quadrants are affected by syndesmophytes in the thoracic spine than in the cervical and lumbar spine, with the highest frequency of syndesmophytes on the right side of the thoracic spine. In the cervical and lumbar spine, most syndesmophytes are found on the anterior side.

Reader 1 scored 453 quadrants with an increase in score of $\geq 1$ between baseline and two years in 44 patients. Reader 2 scored 469 quadrants with an increase in score in 42 patients. Therefore, five patients according to reader 1 and two according to reader 2 did not have any quadrants that increased in score. The mean number of quadrants per patient with an increase in score was 10 for both readers (SD 9 and 10 for reader 1 and 2, respectively). Of the quadrants with an increase in score, 321 were due to new syndesmophytes for reader 1 and 347 for reader 2 . These new syndesmophytes developed in 42 and 43 patients for reader 1 and 2 respectively. Therefore, in 2 patients for reader 1 and 4 patients for reader 2 all quadrants with an increase in score were due to growth of syndesmophytes. Reader 1 scored $37(12 \%)$ new syndesmophytes in the cervical spine, $218(68 \%)$ in the thoracic spine and $66(21 \%)$ in the lumbar spine. Reader 2 scored $72(21 \%)$ new syndesmophytes in the cervical spine, $220(63 \%)$ in the thoracic spine and $55(16 \%)$ in the lumbar spine.

\section{Discussion}

This study describes the development and initial results of the CTSS, a new scoring method for the assessment of syndesmophytes on IdCT in the spine of AS patients. By using a cohort of AS patients with at least 1 syndesmophyte, but not full ankylosis, we have tested the CTSS in a representative sample of AS patients. The CTSS has been shown to have excellent interreader reliability with a status score ICC of 0.99 for the whole spine and 0.97-0.98 for segments of the spine and a similar distribution pattern of scores between readers. Most structural damage was found in the thoracic spine, with approximately two-thirds of patients showing any progression and $30 \%$ showing progression larger than the SDC over two years.

A major advantage of using IdCT for the assessment of syndesmophytes is the ability to scroll through the slices, which gives an unobstructed view on the contours of the vertebrae. Images can be assessed in sagittal and coronal planes, thereby assessing 8 quadrants per VU instead of 2 corners. It was impossible to assess the posterior corners reliably on CR. [9] Furthermore, on IdCT the thoracic spine can be assessed, thereby assessing 24 vertebrae instead of only 12 per patient. The thoracic spine was the section where most structural damage was present and where almost two-thirds of new syndesmophytes were located. Therefore, analyzing this section is very important for the assessment of structural damage. Moreover, on IdCT the facet joints can be assessed as well as the sacroiliac joints, thereby eliminating the need of radiographs of these joints. Another important advantage are the detailed images of IdCT; syndesmophyte height could be scored as more or less than $50 \%$ of the IDS, instead of only as the presence or absence as on mSASSS, which gives the opportunity to assess syndesmophyte growth in more detail. An even more fine-grained assessment of 
syndesmophytes was not feasible, especially in the cervical and upper thoracic spine where deciding between a score of 1 or 2 already proved difficult at times. The change score ICC of the lumbar spine was low, which was caused by minor changes in this segment. The SDC was good, therefore, the low ICC was not due to bad reliability between readers.[10]

A limitation of IdCT compared to radiography is the radiation dose. Low dose CT protocols are currently about a factor 10 lower in dose compared to normal CT but a factor 10 higher in dose compared to radiography, and that was also what we found in a phantom study for this SIAS study.

An effective dose of approximately $4 \mathrm{mSv}$ is received by a patient for a whole spine IdCT. To compare this to radiography, dose values could be derived from the literature. There is a wide variety of reported doses, not always representing current state-of-the art and reported doses may be too high. ((In $2008 \mathrm{CR}$ of the cervical spine $(0.2 \mathrm{mSv})$, thoracic spine $(1.0 \mathrm{mSv})$, lumbar spine $(1.5 \mathrm{mSv})$ and sacroiliac joints (1.5 mSv) combined deliver a radiation dose of $3.4 \mathrm{mSv}$.[11] )) A more realistic estimate was made for our local radiography protocol, using published methodology.[6] This yielded a total dose of $0.4 \mathrm{mSv}$ resulting from cervical and lumbar spine and pelvic radiographs. Background radiation in Europe is approximately $2.6 \mathrm{mSv}$ per year.[12] The European Commission for radiation protection has produced guidelines for radiation exposure in medical research for patients under the age of 50 years.[12] The dose delivered by IdCT falls in category $2 \mathrm{~B}$, which allows research if it is "aimed directly at the diagnosis, cure or prevention of disease". Using IdCT, smaller and possibly more syndesmophytes can be seen compared with CR, therefore, it is likely that earlier identification of progression is possible. This would increase the feasibility of e.g. medication trials for the prevention of progression. Therefore, IdCT fulfills the requirement set by the European Commission. Compared to the background radiation and the added value of IdCT over CR, we consider IdCT a viable method for the assessment of structural damage in the spine.

In summary, the CTSS is a fine-grained scoring system for the assessment of structural damage in the spine of AS patients with excellent interreader reliability. Most structural damage and progression of structural damage was seen in the thoracic spine, the part which is insufficiently assessable on CR. Next step will be to directly compare the assessment of structural damage on IdCT and CR.

Acknowledgements: Dutch Rheumatism Association for providing a grant for the SIAS study. Koos Geleijns, department of radiology, LUMC, Leiden, for performing the phantom studies to estimate the radiation doses.

\section{Conflicts of interest: none}




\section{References}

1. Creemers MC, Franssen MJ, van't Hof MA, et al., Assessment of outcome in ankylosing spondylitis: an extended radiographic scoring system. Ann Rheum Dis 2005;64:127-9

2. Spoorenberg A, de Vlam K, van der Linden S, et al. Radiological scoring methods in ankylosing spondylitis. Reliability and change over 1 and 2 years. J Rheumatol 2004;31:125-32.

3. Tan S, Yao J, Flynn JA, et al. Quantitative measurement of syndesmophyte volume and height in ankylosing spondylitis using CT. Ann Rheum Dis 2014;73:544-50.

4. Tan S, Yao J, Yao L, et al. Improved precision of syndesmophyte measurement for the evaluation of ankylosing spondylitis using CT: a phantom and patient study. Phys Med Biol 2012;57:4683-704.

5. van der Linden, S, Valkenburg HA, Cats A. Evaluation of diagnostic criteria for ankylosing spondylitis. A proposal for modification of the New York criteria. Arthritis \& Rheumatism 1984;27:361-8.

6. Teeuwisse W, Geleijns J, Veldkamp W. An inter-hospital comparison of patient dose based on clinical indications. Eur Radiol. 2007;17:1795-805.

7. Ramiro S, van Tubergen A, Stolwijk C, et al. Scoring radiographic progression in ankylosing spondylitis; should we use the modified Stoke Ankylosing Spondylitis Spine Score (mSASSS) or the Radiographic Ankylosing Spondylitis Spinal Score (RASSS)? Arthritis Res Ther 2013;14:R14.

8. Bruynesteyn K, Boers $M$, Kostense $P$, et al. Deciding on progression of joint damage in paired films of individual patients: smallest detectable difference or change. Ann Rheum Dis 2005;64:179-82.

9. Wanders $A$, Landewé $R$, Spoorenberg A, et al. What is the most appropriate radiologic scoring method for Ankylosing Spondylitis? A comparison of the available methods based on the outcome measures in rheumatology clinical trials filter. Arthritis Rheum 2004;50:2622-32

10. Lukas C, Braun J, van der Heijde D, et al. Scoring inflammatory activity of the spine by magnetic resonance imaging in ankylosing spondylitis: a multireader experiment. J Rheumatol 2007;34:862-70.

11. Mettler FA Jr, Huda W, Yoshizumi TT, et al. Effective doses in radiology and diagnostic nuclear medicine. Radiology 2008;248:254-63.

12. Dutch government, loniserende straling: bronnen en effecten. https://www.autoriteitnvs.nl/onderwerpen/straling/inhoud/ioniserende-stralingbronnen-en-effecten (last access 27 June 2017)

13. Radiation protection 99. Guidance on medical exposures in medical and biomedical research. 1998, European Commission: Brussels, Belgium. p. 1-14. https://ec.europa.eu/energy/sites/ener/files/documents/099_en.pdf (last access 27 June 2017). 